Int. J. Dev. Biol. 50: 675-679 (2006)

doi: $10.1387 /$ ijdb.062167ej

Original Article

\title{
Hox and ParaHox genes in Nemertodermatida, a basal bilaterian clade
}

\author{
EVA JIMÉNEZ-GURI* , JORDI PAPS, JORDI GARCÍA-FERNÀNDEZ\# and EMILI SALÓ\# \\ Departament de Genetica, Facultat de Biologia, Universitat de Barcelona, Barcelona, Spain
}

\begin{abstract}
Molecular evidence suggests that Acoelomorpha, a proposed phylum composed of acoel and Nemertodermatida flatworms, are the most basal bilaterian animals. Hox and ParaHox gene complements characterised so far in acoels consist of a small set of genes, comprising representatives of anterior, central and posterior genes, altogether Hox and ParaHox, but no PG3$X I o x$ representatives have been reported. It has been proposed that this might be the ancestral Hox repertoire in basal bilaterians. However, no studies of the other members of the group, the Nemertodermatida, have been done. In order to get a more complete picture of the basal bilaterian Hox and ParaHox complement, we have analysed the Hox/ParaHox complement of the nemertodermatid Nemertoderma westbladi. We have found representatives of two central and one posterior Hox genes, as well as an XIox and a CaudaIParaHox gene. From our data we conclude that a PG3-XIox gene was present in the ancestor of bilaterians. These findings support the speculation that basal bilaterians already had the beginnings of the extended central Hox set, driving back gene duplications in the central part of the Hox cluster deeper in phylogeny than previously suggested.
\end{abstract}

KEY WORDS: Hox, ParaHox, evolution, nemertodermatida, basal bilateria

\section{Introduction}

The Hox gene family encodes for transcriptional regulators of development, which have a characteristic 60 amino-acid DNA binding motif, encoded by the homeobox. Hox genes play an important role in embryonic development, patterning the A/P axis of the majority of metazoans. This role and the colinearity (whereby the gene order in the genome reflects the antero-posterior pattern of expression in the embryo) they frequently exhibit, predate the divergence of protostomes and deuterostomes (Carroll, 1995). The number and type of Hox genes in a particular animal can be indicative of its phylogenetic relationships (de Rosa et al., 1999). Thus, the presence of a particular gene or a particular peptide motif in one of the genes may be a clue to help assign an animal to one or other bilaterian clade. Several examples of these can be found in the literature, such as the presence of an $a b d-B$ like posterior gene in ecdysozoans whereas Post 1 and 2 genes are found in Lophotrochozoa (de Rosa et al., 1999), the presence of a particular set of central genes in Lophotrochozoa ( $L o x 2,4$ and 5 ) in contrast to those found in Ecdysozoa ( $U b x$ and $a b d-A$ ) (de Rosa et al., 1999), or the presence of "molecular signatures" in some Hox paralogue groups (e.g., "spiralian peptide" in Lox5 or a the "UbdA peptide" at 3'of the Ubx-Lox genes of protostomes, which is not present in deuterostome genes) (Bayascas et al., 1998; de Rosa et al., 1999; Telford 2000; Galant and Carroll, 2002).

The evolution of Hox genes has been the subject of much discussion over the last decade. A consensus of two sister clusters of genes emerging by duplication from a ProtoHox cluster has imposed over other theories (Brooke et al., 1998). These two clusters, Hox and ParaHox, have undergone different patterns of evolution after their split. Briefly, whereas the Hox cluster expanded by tandem duplications during evolution, the ParaHox cluster did not increase the number of genes. Current hypotheses propose a ProtoHox cluster of either 2, 3 or 4 genes (GarciaFernàndez, 2005b). In the 4-gene cluster model, an anterior, a PG3-Xlox, a central and a posterior gene would have given rise to a primordial Hox cluster and a primordial ParaHox cluster of 4 genes. The ParaHox cluster would have then lost the central gene (Brooke et al., 1998); a 3-gene cluster model (with anterior, PG3Xlox and posterior genes) implies the genesis, by tandem duplication, of the central Hox gene in the Hox cluster, after the

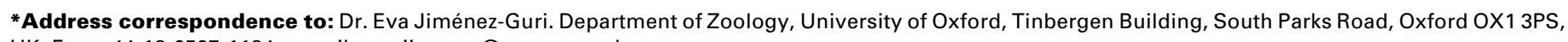
UK. Fax: +44-18-6527-1184. e-mail: eva.jimenez@zoo.ox.ac.uk

\# Note: The indicated authors contributed equally to this work. 
duplication of the ProtoHox cluster (Finnerty and Martindale, 1999; Ferrier and Holland, 2001); the 2-gene cluster model (one anterior, one posterior) implies the genesis, after the ProtoHox cluster duplication, of the PG3 and central Hox genes and of the Xlox ParaHox gene (Garcia-Fernandez, 2005a). The last scenario is based on the absence, so far, of clear Hox PG3 and Central and ParaHox Xlox genes in Cnidarians. Support for the first two hypotheses however comes from branching patterns in phylogenetic trees of the distinct Hox and ParaHox genes, as proposed in Brooke et al. (1998) and Kourakis and Martindale (2000); anterior Hox genes group with Gsx genes, PG3 genes group with Xlox genes and Posterior Hox genes group with $\mathrm{Cdx}$

\begin{tabular}{|c|c|c|c|}
\hline \multicolumn{2}{|c|}{ NweCentralI } & \multicolumn{2}{|l|}{ YNKYLTRRRRIEIAHALNLTERQ } \\
\hline Mye & Scr & $\ldots \ldots \ldots \ldots$ & $\mathrm{AB} 206317.1$ \\
\hline Sro & Central & F.R. . . . . N NL.A. & AY282610.1 \\
\hline Nvi I & $\mathrm{Dfd}$ & F.R.......... & AF151666.2 \\
\hline Mga & $d f d$ & 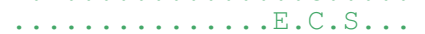 & AJ534451.1 \\
\hline Dti I & Dfd & $\mathrm{F} \ldots \ldots \ldots \mathrm{N}_{\mathrm{N}} \ldots \ldots$ & AJ300661.1 \\
\hline Pfec & $d f d$ & F.R......S.C.A. & AJ272194.2 \\
\hline Pex & Scr & 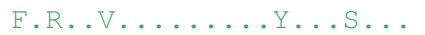 & AY439323.1 \\
\hline Pfe & scr & 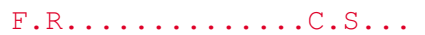 & AJ272190.1 \\
\hline Sca & Scr & F.R. $\ldots \ldots \ldots$. $\ldots$ C & $\mathrm{AF} 393441.1$ \\
\hline Mye 2 & Antp & .R. . . . . . & AB206319.1 \\
\hline Pex $z$ & Antp & .R. . . . & AY439324.1 \\
\hline Nvi & Lox 5 & .R. . W K KG. & AF151671.2 \\
\hline Btu & Lox 5 & R..... & AY497425.1 \\
\hline Alo $z$ & Antp & F.R. . . . . & $\mathrm{AF} 071402.1$ \\
\hline \multicolumn{2}{|c|}{ NweCentralII } & F.H...K...V..S.C. & \\
\hline Mye a & $a b d-A$ & $\mathrm{~F} \cdot \mathrm{H} \ldots \mathrm{K} \ldots \mathrm{V} \ldots \mathrm{S} \cdot \mathrm{C}$. & AB206321.1 \\
\hline DOC & $\mathrm{abd}-\mathrm{A}$ & F.H..K....S.C. & AB206309.1 \\
\hline Pma & $10 \times 4$ & F.H...K...V..S.C. & AJ876626.1 \\
\hline Nvi 1 & Lox 2 & F.R...K...LS.M.C. & AF151668.2 \\
\hline Pex 1 & Lox 4 & F.R..K....C.C. & AY 439327.1 \\
\hline $\mathrm{Csa}$ & ubx & T.H...M.M.S.C. & AJ0 07435.1 \\
\hline Zvi & $\mathrm{Ab} d \mathrm{~A}$ & F.H. . . & AY194829 \\
\hline Pca & Ubx & $\mathrm{F} . \mathrm{H} \ldots \ldots \ldots \mathrm{MSQ}$. C & AF144891.1 \\
\hline Aka & $\mathrm{Ubx}$ & T.H.......... & $\mathrm{AF} 011282.1$ \\
\hline \multicolumn{2}{|c|}{ NweXlox } & \multicolumn{2}{|l|}{ FNKYISRPRRIELAAMLNLTERH } \\
\hline Npo $z$ & Xlox & $\ldots \ldots \ldots \ldots \ldots \ldots \ldots$ & AJ937219.1 \\
\hline Nmi $>$ & Xlox & $\ldots$ & $\mathrm{AF} 363234.1$ \\
\hline Cva $>$ & Xlox & $\ldots \ldots \ldots$ & U68279.1 \\
\hline $\mathrm{Bfl}$ & Xlox & . & $\mathrm{AF} 052464$ \\
\hline Cin $x$ & $\mathrm{Xlox}$ &. $\mathrm{SR} \ldots \ldots \ldots \ldots \ldots$ & AJ296167 \\
\hline Aty $>$ & $\mathrm{Xlox}$ & $\ldots \ldots$ & AF439973.1 \\
\hline \multicolumn{2}{|c|}{ NwePost } & \multicolumn{2}{|l|}{ FNVYITRERRSEISRSLNLTDRQ } \\
\hline Sro & Post & $\ldots{ }_{1} \ldots \ldots \ldots \ldots$ & AY282611.1 \\
\hline Pru & PostB & 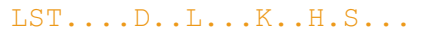 & AY282607.1 \\
\hline Pru & PostA & .N. . . . . . AKV.G.S. . & AY282606.1 \\
\hline Mye & Post1 & NST. .SKS ..W.L.QLI..SE . . & AB206322.1 \\
\hline Pex & Post1 & N.G..S.PE.WHL.CQ . ..E. . & AY439328.1 \\
\hline Nvi & Post2 & G.S. . QK.W..CK.H.SE. . & AF151673.2 \\
\hline Mye & Post2 & NSS . .QK.W. .CK.Q.E.. & AB206323.1 \\
\hline Afr & $\mathrm{AbdB}$ & . . A.VSKQK.W.LA.N. . .E. . & $\mathrm{x} 87250.1$ \\
\hline Lfo $z$ & $\mathrm{AbdB}$ & .A.VSKQK.W.LA.N. ...E. & $\mathrm{AF} 362095.1$ \\
\hline Aka & $A b d B$ & ..A.VSKQK.W.LA.N. . .E. . & $\mathrm{AF} 011274.1$ \\
\hline Bfl & Hox -9 & Y.M.L..... . .QHV ... E & $\mathrm{z} 35149$ \\
\hline \multicolumn{2}{|c|}{ NweCad } & \multicolumn{2}{|l|}{ YKRYLTLRRRVELACELGLTERQ } \\
\hline Nvi & $c d x$ & 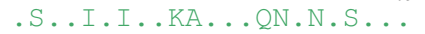 & AY117546.1 \\
\hline Dti & $\mathrm{Cdx}$ & TQK.VNA..KS.M.RA.Q.... & AJ300663.1 \\
\hline Sro & cad & TNQ.I.I..KS . .MQV..S. . & AY282612.1 \\
\hline Pru & cad & TNQ.I.I.KKA...TQV..S . . & AY282608.1 \\
\hline Sma & cad & 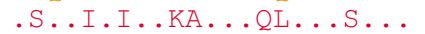 & AY562125.1 \\
\hline Gbi & cad & 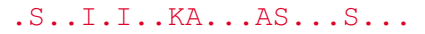 & AB191008.1 \\
\hline Aka c & cad & 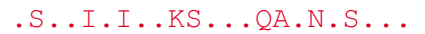 & $\mathrm{AF} 011275.1$ \\
\hline $\mathrm{Xin}$ & cad & TSE.ISTQ.KAY.SRA...S. . & CV579460.1 \\
\hline $\mathrm{Bfl}$ & cad & SNK.I.IK.K.Q..N...S. . & L14866.1 \\
\hline Hro & cad & 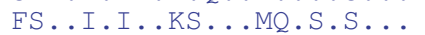 & $\mathrm{AB} 031032.1$ \\
\hline
\end{tabular}

genes. The main caveat of the two-gene ProtoHox theory is that, if it were true, it requires sequence convergence of $\mathrm{Hox} P \mathrm{PG} 3$ and Xlox genes, as those two group together. Therefore, based on sequence parsimony, a 3 or 4 genes ProtoHox cluster is more likely. Starting from these models, Hox genes would have undergone several tandem duplications, increasing the complexity of the cluster during evolution, whereas ParaHox genes would not have increased in number (in the 3-gene model) or would have lost the central class gene (in the 4-gene model).

The Acoelomorpha flatworms have been proposed to be those most basal bilaterian animals and the outgroup of protostomes plus deuterostomes (Ruiz-Trillo et al., 2002, Jondelius et al., 2002, Telford et al., 2003). This clade comprises two groups: the Acoela and the Nemertodermatida. There has been a debate about the monophyly of this group: 18S data suggests paraphyly of the group (Jondelius et al., 2002), but analysis of myosin heavy chain II sequences (Ruiz-Trillo et al., 2002) and the mitochondrial genome (Ruiz-Trillo etal., 2004), plus morphological data strongly support monophyly of the group (Baguñà and Riutort, 2004).

A small set of Hox and ParaHox genes have been found so far in acoel flatworms: one anterior, one central and one posterior Hox gene and only a posterior ( $C d x$ ) ParaHox gene (Cook et al., 2004). It was proposed, in the light of this data, that the primitive bilaterian had no representative of PG3-Xlox genes (Cook et al., 2004). This conclusion was based only on one of the groups of the Acoelomorpha. Studies in the smaller sister group, the Nemertodermatida, are thus important for clarification of the basal bilaterian condition of the Hox and ParaHox complements.

In this work, we report PCR-generated sequences of Hox and ParaHox genes in the nemertodermatid Nemertoderma westbladi which improve our understanding of the complements of these genes in the sister group of the eubilaterians. We demonstrate the presence of a Group3/Xlox ParaHox gene prior to the protostome/ deuterostome divergence.

\section{Results}

In order to get an accurate view on basal bilaterian Hox and ParaHox content, we performed PCR on the Nemertodermatida Nemertoderma westbladiusing degenerate primers which coded

Fig. 1. Alignment of the genes of Nemertoderma westbladi (black font) isolated, with other bilaterian orthologs (red for Ecdysozoa, green for Lophotrochozoa and yellow for Acoela). Species names abbreviated as follows. Afr, Artemia franciscana. Aka, Acanthokara kaputensis. Alo, Archegozetes longisetosus. Aty, Archaster typicus. Bfl, Branchiostoma floridae. Btu, Bugula turrita. Cin, Ciona intestinalis. Csa, Cupiennius salei. Cva, Chaetopterus variopedatus. Doc, Dentalium octangulatum. Dti, Discocelis tigrina. Gbi, Gryllus bimaculatus. Hro, Halocynthia roretzi. Lfo, Lithobius forficatus. Mga, Mytilus galloprovincialis. Mye, Mizuhopecten yessoensis. Nmi, Nephasoma minuta. Npo, Nautilus pompilius. Nvi, Nereis virens. Pca, Priapulus caudatus. Pex, Perionyx excavatus. Pfe, Pachymerium ferrugineum. Pma, Pecten maximus. Pru, Paratomella rubra. Sca, Sacculina carcini. Sma, Strigamia maritime. Sro, Symsagittifera roscofensis. Xin, Xiphinema index. Zvi, Zaprionus vittiger. Dots denote amino acid identity of a particular gene to the Nemertoderma westbladi gene directly above. All Nemertoderma westbladi genes shown have unique nucleotide sequences when compared to the NCBI database, ruling out contamination from any known Hox or ParaHox gene. Orthology has been assigned by blastn best hit. 

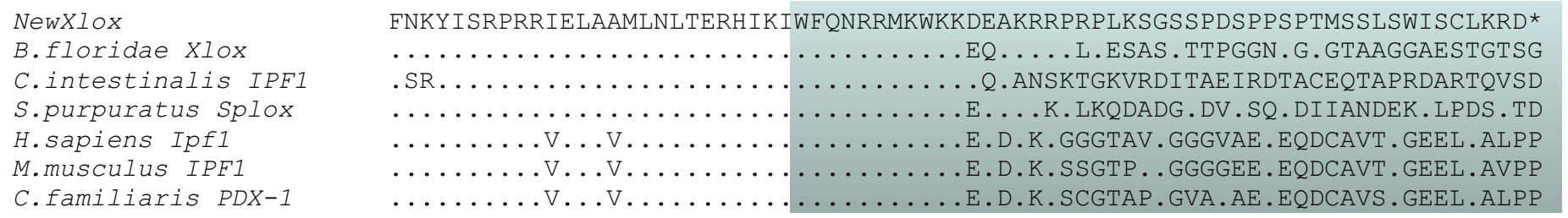

Fig. 2. Comparison of the partial NweXIox sequence that extends 3'of the homeobox (shadowed box is the RACE extended sequence). Dots denote identity and bars have been placed where no sequence is available. Lack of Xlox sequence information outside the homebox in other groups does not allow phylogenetic analysis with this part of the gene. No 100\% nucleotide similarity sequence was found in the database, even for the shorter helix1-helix3 sequence. Sequences used are: B. floridae AAC39016, C. intestinalis AJ296167, S. purpuratus NP_999815.2, H. sapiens NP_000200.1, M. musculus CAA52389.1, C. familiaris XP_543155.2.

for the helix1 and helix3 regions of the homeodomain. The PCR reactions yielded fragments of approximately $115-120 \mathrm{bp}$, which were subsequently cloned. Sequences of 126 homeobox-containing clones identified a total of five distinct sequences with similarity to particular paralogous Hox and ParaHox groups (PG, genes related by cluster duplication; we will subsequently refer to the gene groups by the deuterostome nomenclature for naming simplification purposes) (Fig. 1). It is plausible that some other Hox or ParaHox genes were missed by our approach, due to sequence divergence or lack of expression in the RNA sample used for the PCR. To minimise the number of genes missed by PCR we used, in addition to general homoebox-degenerated primers, a set of specific primers targeting the different PGs. Nevertheless, in the sister group of Nemertodermatida, the Acoela, a similar number of genes was found (Cook et al., 2004), but noticeably not a representative of the PG3/Xlox gene. We have named the genes with the prefix Nwe (for Nemertoderma westblad) followed by a name that characterises each paralogy group. We have found two clearly distinct central Hox genes (NweCentral-/ and NweCentral-II), one posterior Hox gene (NwePost), an Xlox ParaHox gene (NweX/ox) and a Caudal ParaHox gene (NweCad). Further sequence of the NweXlox was obtained using a gene specific primer and a 3' SMART adaptor primer as described in the M\&M section. Sequences were submitted to GenBank under accession numbers DQ677343-DQ677347.

Two of the genes found are representatives of the central class Hox genes (NweCentral-/ and NweCentral-II). On the basis of sequence similarity, NweCentral-// is more closely related to central Hox genes of the PG6-8 group, whereas NweCentral-I cannot be assigned to any particular paralogy group. It has been proposed that at the base of bilaterians there was, at the most, one central gene (Garcia-Fernàndez, 2005a). Nemertodermatida have now been found to have two clearly distinct central genes. We propose, in the light of our findings plus the data previously available from Acoela (Cook et al., 2004), that Acoelomorpha have two central genes, one which would group with PG4/5 and a second one that would group with PG6-8. Those genes in the base of the bilaterian clade could have given rise to the set of central Hox genes seen in Eubilateria.

We have also cloned a posterior Hox gene (NwePost). Blast hits classify NwePostamongst PG10. Posterior lophotrochozoan and ecdysozoan Hox genes differ in sequence and number. Lophotrochozoans possess two posterior Hox genes, whereas ecdysozoans only have one type of posterior gene; they are distinguishable by several diagnostic residues within the helix 1 - helix3 regions. Deuterostomes have an extended posterior gene complement, also with some diagnostic residues. Therefore, orthology assignment to the posterior genes is usually relatively easy. Nevertheless, as for Acoela (Cook et al., 2004), Nemertodermatida do not share any of the diagnostic residues for posterior Hox genes with any of the three major bilaterian clades, as expected from its position as an outgroup of eubilaterians. Trees built with this short sequence, even though they do not have good bootstrap values, do indeed support the lack of clear relationship to a particular higher bilaterian clade as well (data not shown). Acoela and Nemertodermatida posterior genes are not extremely similar, but share some amino-acid residues that differ from posterior genes of other groups. We suggest that the existence of a single posterior Hox gene in Acoelomorpha is a prototypic feature, even though its sequence has diverged and evolved differently in Acoela and Nemertodermatida after these groups split.

Of the ParaHox genes, a posterior caudal gene was identified (NweCad) and, contrary to the model of Cook etal. (2004), an XIox representative was found (NweXlox). The NweCadgene does not possess some residues that have been until now considered typical for Cad proteins, but in BLAST searches, it gives consistently higher homology to the caudal group than to any other gene. NweXIox was further elongated by RACE, yielding a longer sequence at the 3 ' end of the fragment (Fig. 2). The finding of a NweXIox gene challenges and changes the proposed ideas for Acoelomorpha Hox and ParaHox complements. Acoelomorpha were thought to lack representatives of the PG3/Xlox genes. Our work has shown they have indeed representatives of all 4 major Hox classes and therefore at the base of bilaterians those classes were present.

\section{Discussion}

We have cloned two central Hox genes in Nemertodermatida: NweCentral-/and NweCentral-II. We have classified NweCentral/as related to PG6-8 whereas NweCentral-//cannot be assigned unequivocally to a particular paralogy group. Several hypotheses may account for the presence of these two distinct genes. First, this could be due to an independent duplication in the Nemertodermatida, after the separation from Acoela, from a single ancestral central gene. In this case, Nemertodermatida would have evolved a PG6-8-like gene and acoels evolved a PG4/ 5-like gene (Cook et al., 2004) from a single, unique ancestral Central (which would be the ancestor of PG $4 / 5$ and PG6-8 genes 


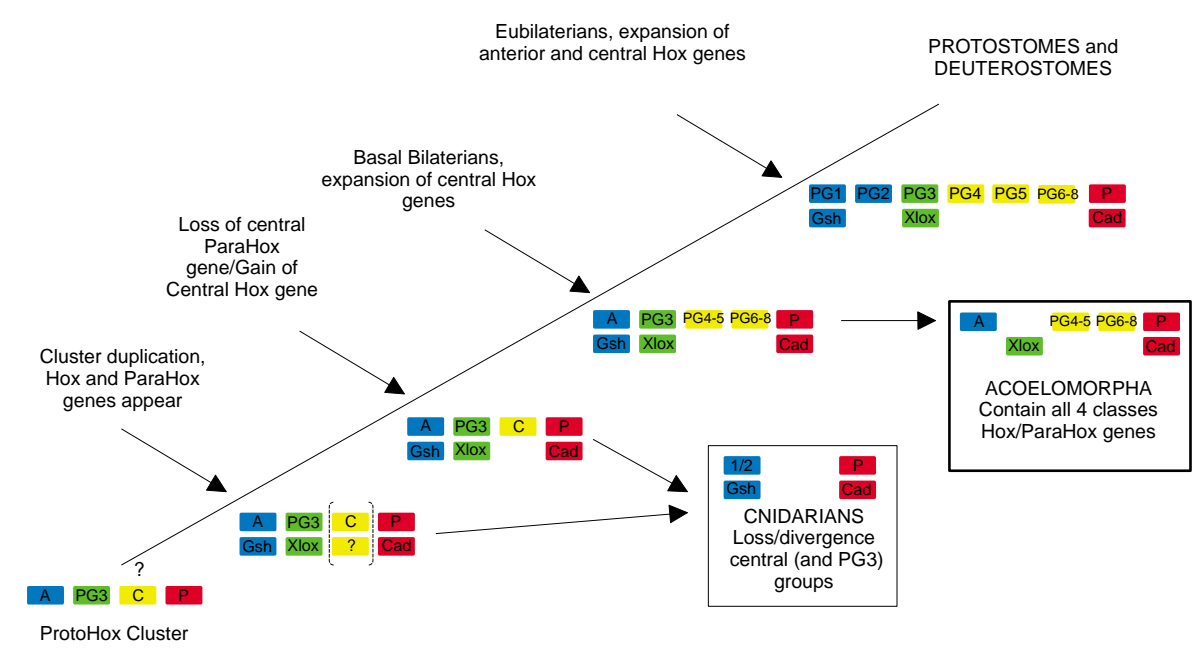

Fig. 3. Proposed evolution of Hox and ParaHox genes starting from the 4-gene ProtoHox cluster (Brooke et al., 1998) or a 3-gene ProtoHox cluster (question mark and dashed parenthesis). Basal bilaterians show a reduced set of genes, but an expansion of the central Hox genes, which could have been related to the acquisition of bilaterian features. All four ProtoHox gene classes are found in Acoelomorpha.

of Eubilateria). This would necessarily imply that Nemertodermatida NewCentral-//and Eubilateria PG6/8 are similar by convergent evolution and that Acoela SrHox4/5 and Eubilateria PG4/5 are similar by convergent evolution. We find this very unlikely and propose, instead, that Acoelomorpha ancestrally had at least two central genes, one PG4-5 and one PG6-8 and that later evolution led to the loss of the PG6-8 gene in Acoela and of the PG4-5 gene in Nemertodermatida. Subsequently, in Nemertodermatida there was a duplication of the central gene. Another possibility that cannot be ruled out is that Acoelomorpha had ancestrally 3 central genes and two have been kept in Nemertodermatida and one in Acolea (or are yet to be found in each group). Nevertheless, we think NweCentral-/is an independent duplication in Nemertodermatida as it has no clear similarity to any other central gene in any phyla.

It has been proposed that the extension of central Hox genes led to the expansion of the higher bilaterians at the time of the Cambrian Explosion (Garcia-Fernandez 2005a, 2005b for latest reviews). From our data, we suggest that the duplication of central Hox genes began earlier in evolution. Hence, the appearance of complex eubilaterians in the Cambrian Explosion would have coincided with less extensive expansion of central Hox genes than previously believed.

We have found a single posterior Hox gene in Nemertodermatida (NwePost). This gene is different from the Acoela posterior gene described by Cook et al., even though they share some residues that are not present in the other groups. Nevertheless, neither NwePost nor the acoela posterior genes share any diagnostic residues with any of the existing eubilaterian posterior Hox genes. We suggest that the existence of a single posterior Hox gene in Acoleomorpha is a prototypic feature, even though the posterior gene has diverged and evolved in both groups after their split.

We have found two ParaHox genes in Nemertodermatida:
NweCad (posterior ParaHox gene) and NweXlox (XIoxrepresentative). Finding the $X$ Iox representative changes the vision for the Hox and ParaHox complement in Acoelomorpha. We can now be certain that in Acoelomorpha there is an X/oxrepresentative and therefore, given the Acoelomorpha are indeed basal bilaterians, we propose that there were representatives of all 4 classes of Hox genes at the base of the bilaterian lineage (Fig. 3). If that is the case, the model proposed by Ferrier and Holland (2001) (4 distinct classes of Hox genes existed before the divergence of cnidarians and bilaterians) can still be considered, as it is not contradicted by Acoelomorpha data, although still implies losses in Cnidarians, whereas most importantly, the proposed scenario of Cook et al. (2004), in which the Xlox/PG3 genes appeared after the origin of the Bilateria must be discarded.

In summary, we speculate, based on the data available and assuming that Acoelomorpha is a monophyletic basal bilaterian group, that the beginning of an extended central Hox set was present at the base of bilaterians. Also, we propose that basal bilaterians had at least all 4 ProtoHoxclasses derived genes (and probably five, including two central genes). Our data cannot help in distinguishing which of the 2,3 or 4 gene ProtoHox cluster model is correct (as the ProtoHox cluster duplication occurred before the cnidarian/bilaterian split), but indicates that in the separate branches within the sister group (Acoelomorpha) to the Eubilateria there may have been differential gene loss of certain groups of genes. In Acoelomorpha as a whole we can find at least one gene of each class (Anterior, Group3/Xlox, CentralPG4/5, Central PG6/8 and Posterior) when taking into account both Hox and ParaHox genes, including an $X / o x$ representative. We believe that our model for the early evolution of bilaterian Hox/ParaHox clusters, for which caveats derived from PCR screenings and short sequences cannot be discarded, will prompt further research to clarify the long-standing doubt cast on the early function and evolution of the paradigmatic Hox gene family.

\section{Materials and Methods}

Adults of Nemertoderma westbladi were collected near Kristineberg Marine Research Station (Sweden), immediately immersed in Trizol reagent (Sigma) and kept at $4^{\circ} \mathrm{C}$. To obtain $\mathrm{RNA}$, the protocol for the Trizol Reagent (Sigma) was used according with the manufacturer's specifications. cDNA was obtained by using the SMART cDNA library construction kit (Clontech), after 24 rounds of amplification. This process links specific flanking regions (SMART adaptors) to the DNA, different at the 5' and 3' ends. This cDNA was used as template for PCR amplification using degenerated primers.

A first round of PCR was preformed using primers for the 3' (5'ATTCTAGAGGCCGAGGCGGCCGACATG-3') or 5' (5'AAGCAGTGGTATCAACGCAGAGT-3') SMART adaptors combined with degenerate primers of the regions of the helix1 (ELEKEF, QLELE, 
YQTLELEK, LELEKE) or the helix3 (WFQNRR, KIWFQN, FQNRR, QVKIWF, QIKIWF) of the homeodomain, respectively. Different sets of primers were used to target classes of different Hox genes. A semi-nested PCR was then performed on the PCR products with the helix 1 and helix 3 degenerate primers.

The 115-120 bp PCR products obtained were cloned in pSK Bluescript, transformed in $E$. coli JM105 cells and plated on selective medium. Selection of insert containing colonies was done using blue-white screening. DNA minipreparation of single white colonies was preformed using the QIAprep Spin Miniprep kit (Qiagen). Sequence of the clones was performed with M13F primer using a $\mathrm{ABI} /$ Prism37100 sequencer. The sequences obtained were compared to the GenBank database using blastn. Anchored PCR of those sequences was performed using specific gene primers and the SMART adaptors primers on all the genes found, although it worked only for the NweXIox fragment. Alignments of the sequences were performed using ClustalX.

\section{Acknowledgments}

E.J.G. would like to thank Ellard Keith, Thomas Butts and Karl Wotton for comments on the manuscript and the Oxford Evo-Devo group for helpful discussion on different stages of the manuscript. We would like to thank three anonymous reviewers for their suggestions. This work was supported by the funds from the Ministerio de Educación y Ciencia, Spain, PB98-1261-C02-01. E.J.G. was funded by a BRD from the Universitat de Barcelona.

\section{References}

BAGUÑÀ J, RIUTORT M (2004) The dawn of bilaterian animals: the case of acoelomorph flatworms. BioEssays 26: 1046-1057.

BAYASCAS JR, CASTILLO E, SALO E (1998) Platyhelminthes have a Hox code differentially activated during regeneration, with genes closely related to those of spiralian protostomes. Dev Gen Evo/208: 467-473.

BROOKE NM, GARCIA-FERNANDEZ J, HOLLAND PWH. (1998) The ParaHox gene cluster is an evolutionary sister of the Hox cluster. Nature 392: 920-922.

CARROLL SB (1995) Homeotic genes and the evolution of arthropods and chordates. Nature 376: 479-485.
COOK CE, JIMÉNEZ E, AKAM M, SALO E (2004) The Hox gene complement of acoel flatworms, a basal bilaterian clade. Evol Dev. 6: 154-63.

DE ROSA R, GRENIER JK ANDREEVA T, COOK CE, ADOUTTE A, AKAM M, CARROLL SB, BALAVOINE G (1999) Hox genes in brachiopods and priapulids and protostome evolution. Nature. 399: 772-6

FERRIER DEK, HOLLAND PWH (2001) Ancient origin of the Hox gene cluster. Nat Rev Genet 2: 33-38

FINNERTY JR, MARTINDALE MQ (1999). Ancient origins of axial patterning genes: Hox genes and ParaHox genes in the Cnidaria. Evol Dev 1: 16-23

GALANT R, CARROLL SB (2002) Evolution of a transcriptional repression domain in an insect Hox protein. Nature. 415: 910-3.

GARCIA-FERNANDEZ J (2005a) Hox, ParaHox, ProtoHox: facts and guesses. Heredity. 94: 145-52.

GARCIA-FERNANDEZ J (2005b) The genesis and evolution of homeobox gene clusters. Nature Rev.Genetics 6: 881-892.

JONDELIUS U, RUIZ-TRILLO I, BAGUÑÀ J, RIUTORT, M (2002) The nemertodermatid flatworms are basal bilaterians not members of Platyhelminthes. Zool SCr 31: 201-215

KOURAKIS MJ, MARTINDALEM.Q. (2000) Combined-method phylogenetic analysis of Hox and ParaHox genes of the metazoa. J Exp Zool 288: 175-191

RUIZ-TRILLO I, PAPS J, LOUKOTA M, RIBERA C, JONDELIUS U, BAGUÑ̇̀ J, RIUTORT M (2002) A phylogenetic analysis of myosin heavy chain type II sequences corroborates that Acoela and Nemertodermatida are basal bilaterians. Proc Natl Acad Sci USA. 99: 11246-11251

RUIZ-TRILLO I, RIUTORT M, FOURCADE HM, BAGUÑÀ J, BOORE JL (2004) Mitochodrial genome data support the basal position of Acoelomorpha and the polyphyly of the Platyhelminthes. Molec. Phylogen. Evol. 33: 321-332.

TELFORD MJ (2000) Turning Hox "signatures" into synapomorphies. Evol Dev2: 360-364

TELFORD MJ, LOCKYER AE, CARTWRIGHT-FINCH C, LITTLEWOOD DT (2003) Combined large and small subunit ribosomal RNA phylogenies support a basal position of the acoelomorph flatworms. Proc Biol Sci. 270: 1077-83.

Received: 30th March 2006 Reviewed by Referees: 4th May 2006 Modified by Authors and Accepted for Publication: 29th June 2006 Published Online: 3rd October 2006 